\title{
Mécanismes d'endommagement dans les matériaux composites à matrice d'alliage de titane renforcée par des filaments de carbure de silicium
}

\author{
ÉRIC MARTin ${ }^{1, a}$ ET NiCOlas CARRÈre ${ }^{2}$ \\ 1 Laboratoire des Composites Thermostructuraux, UMR 5801 (CNRS - UB1 - SNECMA-CEA), Domaine Universitaire, \\ 3 allée de la Boétie, 33600 Pessac, France \\ 2 ONERA, DMSE, 29 avenue de la Division Leclerc, 92322 Châtillon Cedex, France
}

Reçu le 10 juin 2003, accepté le 7 novembre 2003

\begin{abstract}
Résumé - Les hautes propriétés spécifiques des matériaux composites à matrice d'alliage de titane renforcée unidirectionnellement par des filaments de carbure de silicium permettent d'envisager leur emploi pour des applications aéronautiques. Après une brève description des constituants et du mode d'élaboration, les principaux mécanismes d'endommagement sont présentés. Les ruptures de filaments sont observées dans le cas d'une sollicitation longitudinale tandis que la rupture de l'interface est mise en évidence dans le cas d'une sollicitation transverse. Diverses approches multiéchelles destinées au dimensionnement de pièces en composite $\mathrm{SiC} / \mathrm{Ti}$ sont ensuite envisagées. Une analyse basée sur la description du mécanisme de rupture des filaments permet d'estimer la contrainte à la rupture dans le cas d'une sollicitation longitudinale. Une méthode de transformation de champ permet d'obtenir une loi de comportement prenant en compte les contraintes résiduelles de fabrication. Elle est utilisée pour le calcul d'un disque de compresseur de turboréacteur renforcé circonférentiellement par un composite $\mathrm{SiC} / \mathrm{Ti}$.
\end{abstract}

Mots clés : Composites $\mathrm{SiC} / \mathrm{Ti}$ / mécanismes d'endommagement / interface filament/matrice / approche multiéchelle / calcul de structure

\begin{abstract}
Damage mechanisms in SiC/Ti composites. Titanium matrix composites made with a titanium alloy matrix reinforced with silicon carbide filaments have demonstrated potential for propulsion applications because of their excellent specific properties. Possible applications include hoop reinforced components such as bladed rings. Damage mechanisms in such composite materials are presented as revealed by mechanical testing. Filament fracture is observed in the case of longitudinal loading while interface fracture is dominant in the case of transverse loading. Various multiscale analysis can be used to predict the mechanical behaviour of $\mathrm{SiC} / \mathrm{Ti}$ composites. For a longitudinal loading, an approach based on the description of the development of filament fractures allows to evaluate the fracture stress of the composite. A transformation field method is used to derive a constitutive law of the composite which takes into account the residual stresses after elaboration. In this case, computation results concerning a disk reinforced by a composite ring are provided.
\end{abstract}

Key words: $\mathrm{SiC} / \mathrm{Ti}$ composites / damage mechanisms / filament/matrix interface / multiscale approach / structural analysis

\section{Introduction}

Le développement des matériaux composites à matrice d'alliage de titane renforcée par des filaments céramiques en carbure de silicium ( $\mathrm{SiC} / \mathrm{Ti}$ ) est motivé par leurs bonnes propriétés spécifiques dans le sens du renfort

\footnotetext{
a Auteur correspondant : martin@lcts.u-bordeaux.fr
}

jusqu'à des températures proches de $500{ }^{\circ} \mathrm{C}$ [1]. Plusieurs applications aéronautiques comme des arbres de transmission et des pales de compresseurs sont envisagées. Cependant, la principale application à court terme concerne leur utilisation dans les disques de compresseur de turboréacteur [2]. L'orientation des renforts dans un anneau aubagé monobloc (Fig. 1) permet de reprendre les efforts circonférentiels de la pièce tournante et d'alléger de plus 


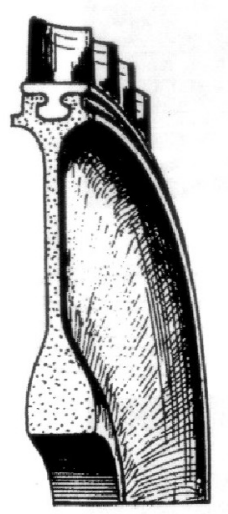

Référence

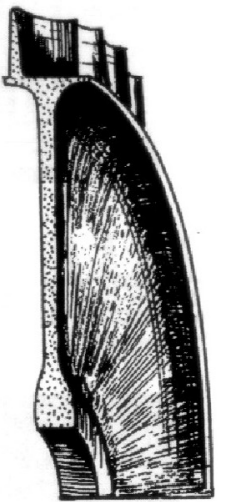

gain de masse $35 \%$

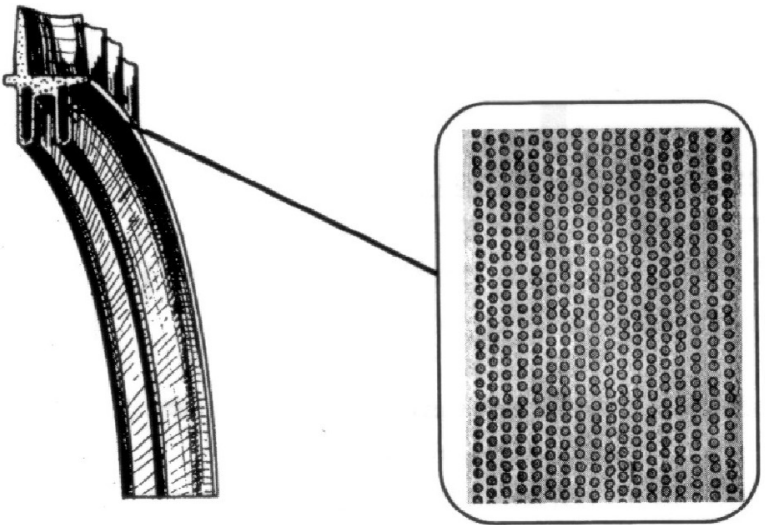

gain de masse

$55 \%$

Fig. 1. Renforcement d'un disque de compresseur par un anneau en composite SiC/Ti.

de $50 \%$ la masse du disque. Le dimensionnement de cet anneau nécessite la compréhension des mécanismes d'endommagement pouvant conduire à la ruine du matériau composite.

L'objectif de ce papier est la présentation de ces mécanismes et de certaines approches multiéchelles permettant de dimensionner une pièce en composite $\mathrm{SiC} / \mathrm{Ti}$. $\mathrm{Au}$ préalable, une rapide description des divers constituants du matériau composite ainsi que de son mode d'élaboration est nécessaire.

\section{Le matériau composite $\mathrm{SiC} / \mathrm{Ti}$}

La figure 2 est une micrographie de la section d'un matériau composite $\mathrm{SiC} / \mathrm{Ti}$. On y distingue des filaments de carbure de silicium renforçant unidirectionnellement une matrice d'alliage de titane.

\subsection{Le renfort et la matrice}

Le tableau 1 présente les caractéristiques mécaniques des différents filaments utilisés dans ces matériaux. Ce renfort est produit par dépôt chimique en phase vapeur de carbure de silicium sur une âme en carbone de diamètre $33 \mu \mathrm{m}$ (cas des filaments SCS d'un diamètre d'environ $140 \mu \mathrm{m}$ ) ou en tungstène de diamètre $15 \mu \mathrm{m}$ (cas des filaments SM d'un diamètre d'environ $100 \mu \mathrm{m}$ ). La microstructure de ces filaments est complexe et formée de plusieurs couches de morphologies différentes [3]. Il a été montré que l'interface âme/SiC est un site privilégié d'amorçage lors de la rupture du filament [4]. On notera la présence d'un revêtement externe (d'une épaisseur de quelques microns) en carbone principalement destiné à protéger le filament lors de la phase de fabrication [5].

Les alliages de titane sont habituellement classés en différentes catégories suivant les proportions respectives en phase $\alpha$ et $\beta$. Différents alliages de titane ont déjà été utilisés pour la fabrication du composite (Tab. 2).

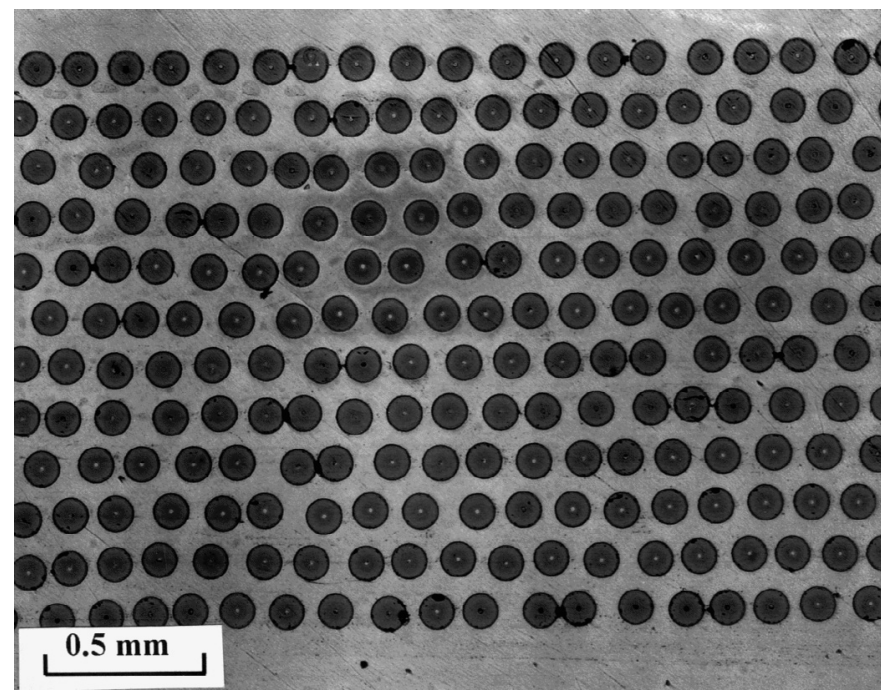

Fig. 2. Section d'un composite $\mathrm{SiC} / \mathrm{Ti}$.

Tableau 1. Caractéristiques mécaniques de quelques filaments de carbure de silicium à température ambiante $\left(\sigma^{\mathrm{R}}\right.$ et $\varepsilon^{\mathrm{R}}$ sont respectivement la contrainte et la déformation à la rupture) [22].

\begin{tabular}{cccccc}
\hline Filament & $\begin{array}{c}\text { Diamètre } \\
(\mu \mathrm{m})\end{array}$ & $\begin{array}{c}\mathrm{E} \\
(\mathrm{GPa})\end{array}$ & $\begin{array}{c}\alpha \\
\left(10^{-6}{ }^{\circ} \mathrm{C}^{-1}\right)\end{array}$ & $\begin{array}{c}\varepsilon^{\mathrm{R}} \\
\%\end{array}$ & $\begin{array}{c}\sigma^{\mathrm{R}} \\
(\mathrm{MPa})\end{array}$ \\
\hline SM1140+ & 108 & 400 & 4 & 1 & 3300 \\
SCS6 & 143 & 400 & 3 & - & 3800 \\
\hline
\end{tabular}

La modification des éléments d'addition permet d'augmenter la température d'utilisation [6].

\subsection{Les modes d'élaboration}

Le matériau composite est élaboré à partir d'une préforme constituée d'un empilement de nappes de fibres et de feuillards d'alliage ou de nappes de filaments 

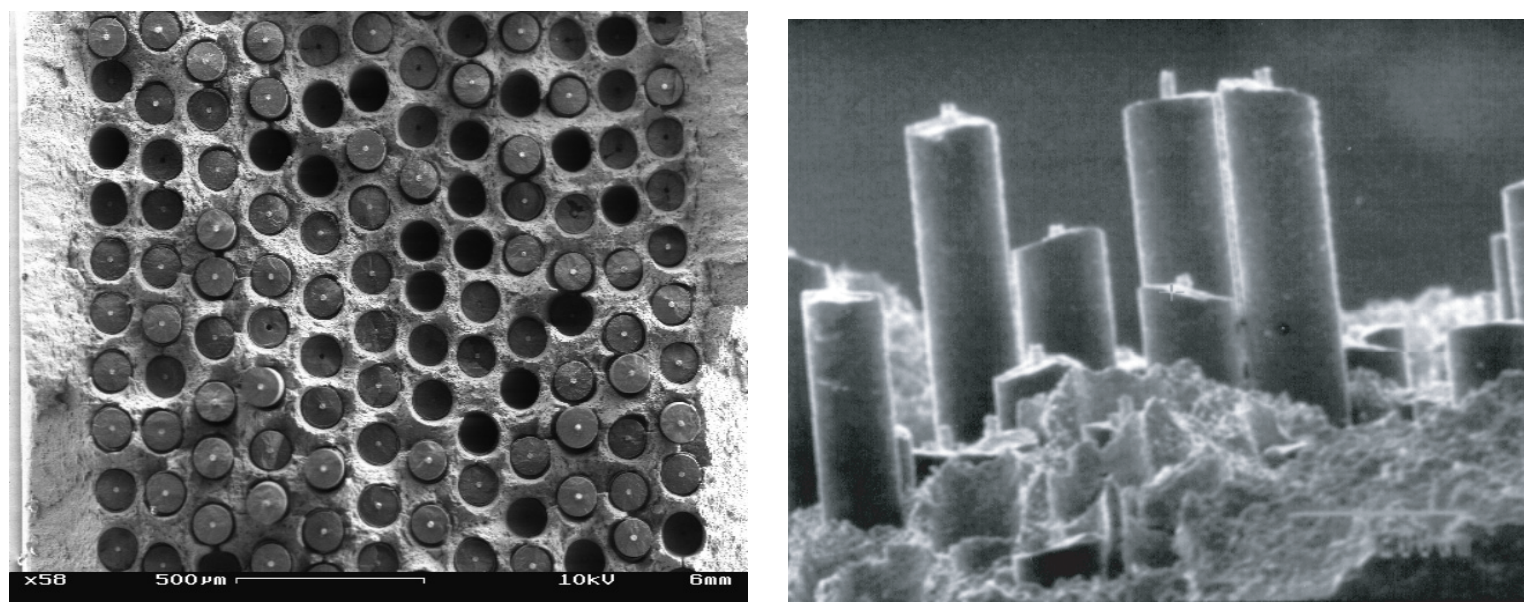

Fig. 3. Observations fractographiques sur une éprouvette de composite $\mathrm{SiC} / \mathrm{Ti}$ sollicitée dans la direction longitudinale.

Tableau 2. Caractéristiques mécaniques de quelques alliages de titane à température ambiante $\left(\sigma^{\mathrm{R}}\right.$ et $\sigma^{y}$ sont respectivement la contrainte à la rupture et la limite d'élasticité à $0,2 \%)[22]$.

\begin{tabular}{lccccc}
\hline Alliage & $\begin{array}{c}\mathrm{E} \\
(\mathrm{GPa})\end{array}$ & $\begin{array}{c}\alpha \\
\left(10^{-6}{ }^{\circ} \mathrm{C}^{-1}\right)\end{array}$ & $\begin{array}{c}\varepsilon^{\mathrm{R}} \\
\%\end{array}$ & $\begin{array}{c}\sigma^{\mathrm{R}} \\
(\mathrm{MPa})\end{array}$ & $\begin{array}{c}\sigma^{\mathrm{y}} \\
(\mathrm{MPa})\end{array}$ \\
\hline Ti-6-4 & 110 & 9,5 & $13-16$ & 925 & 870 \\
Ti-6242 & - & - & 12 & 1020 & 900 \\
Ti-15-3 & 90 & - & $10-20$ & $800-1000$ & - \\
$\beta 21 \mathrm{~S}$ & 80 & - & 7 & 1400 & 1300 \\
\hline
\end{tabular}

revêtues de matrice par dépôt plasma ou pulvérisation cathodique d'alliage de titane [7]. Ensuite, la consolidation s'effectue à l'état solide sous haute température et haute pression par pressage isostatique ou compression uniaxiale. On notera cependant que la diminution du coût d'élaboration du matériau nécessite le développement de nouveaux procédés pour obtenir un filament revêtu d'une gaine de matrice [8]. Un procédé d'enduction métallique des filaments par voie liquide a été développé récemment [9].

Ce mode d'élaboration à haute température provoque au niveau de l'interface filament/matrice la formation d'une zone de réaction possédant une épaisseur de l'ordre du micron. La nature et la microstructure de cette interphase dépendent de la matrice et du revêtement du filament. Les produits de cette réaction interfaciale sont en général des composés fragiles formés de carbures et de siliciures de titane ainsi que d'éléments d'alliage [10].

Compte tenu de la différence entre les coefficients de dilatation du renfort et de la matrice, la phase de refroidissement qui suit la fabrication à haute température du composite génère des contraintes résiduelles dans le composite. L'amplitude de ces contraintes peut être évaluée expérimentalement par dissolution matricielle [11], ou à l'aide d'analyse par tomographie X [12] et estimée numériquement à l'aide d'une modélisation par éléments finis en prenant en compte le comportement viscoplastique de la matrice [13]. À température ambiante et lorsque la fraction volumique de renfort est de l'ordre de $35 \%$, les résultats montrent que la matrice est en tension axiale $(\approx 400 \mathrm{MPa})$, le renfort est en compression axiale $(\approx 900 \mathrm{MPa})$ tandis que l'interface est en compression radiale $(\approx 300 \mathrm{MPa})$. Cet état de contraintes initial ne peut être ignoré lors de l'étude des mécanismes d'endommagement.

\section{Endommagement du $\mathrm{SiC} / \mathrm{Ti}$}

Les essais mécaniques sur composite $\mathrm{SiC} / \mathrm{Ti}$ nécessitent des procédures particulières pour éviter les concentrations de contrainte qui provoquent des ruptures hors de la zone utile $[14,15]$.

\subsection{Sollicitation longitudinale}

Les essais de traction dans le sens longitudinal mettent en évidence les ruptures des filaments qui sont bien détectées par émission acoustique car elles produisent des événements de forte amplitude [16]. Les examens fractographiques (Fig. 3) indiquent que ces ruptures s'accompagnent de décohésion interfaciale entre la zone de réaction et le dépôt de carbone externe sur des longueurs pouvant atteindre deux à trois diamètres de filament. Des observations en cours d'essai par tomographie X ont montré que les ruptures de filaments étaient couplées : la rupture d'un filament provoque la surcharge des filaments voisins qui rompent à leur tour [12]. Le tableau 3 indique que la déformation à rupture qui est contrôlée par les filaments est de l'ordre de $1 \%$.

\subsection{Sollicitation transversale}

La figure 4 montre l'allure d'une courbe de traction dans le sens transverse. La rupture de l'interface entre 


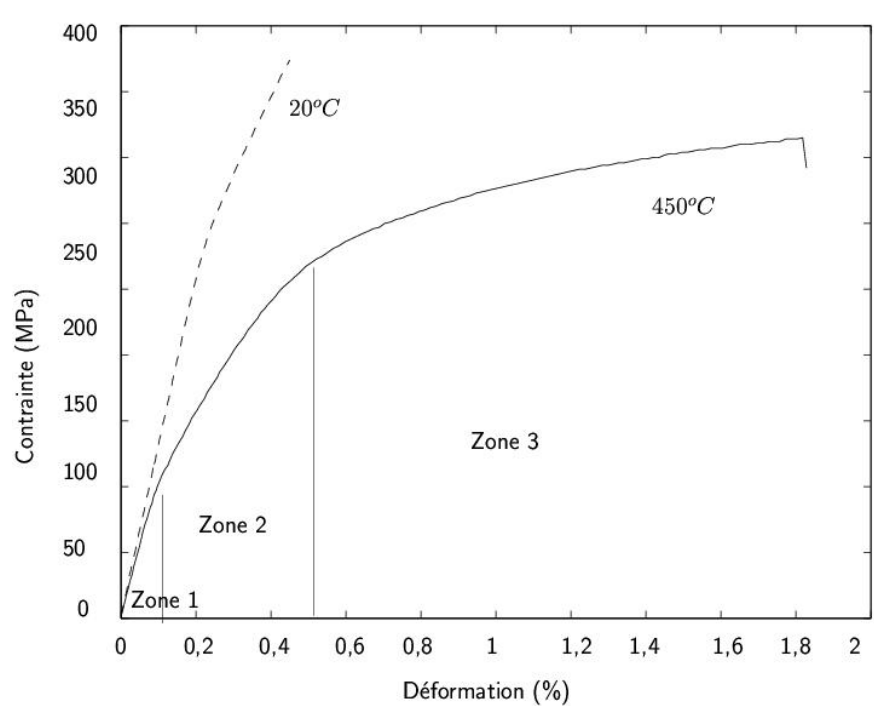

Fig. 4. Courbes de traction pour une éprouvette de composite $\mathrm{SiC} / \mathrm{Ti}$ sollicitée dans la direction transverse.

Tableau 3. Caractéristiques mécaniques d'un composite SM1140+/Ti-6-4 (fraction volumique de renfort : $33 \%$ ) à température ambiante $\left(\sigma^{\mathrm{R}}\right.$ et $\varepsilon^{\mathrm{R}}$ sont respectivement la contrainte à la rupture et la déformation à la rupture) [26].

\begin{tabular}{llll}
\hline Orientation & $\mathrm{E}$ & $\sigma^{\mathrm{R}}$ & $\varepsilon^{\mathrm{R}}$ \\
& $(\mathrm{GPa})$ & $(\mathrm{MPa})$ & $\%$ \\
\hline Longitudinale & 200 & 1680 & 1,1 \\
Transverse & 115 & 155 & 0,9 \\
\hline
\end{tabular}

la zone de réaction et le dépôt de carbone externe provoque une perte de linéarité à l'issue de la première partie de la courbe. Cet endommagement se propage durant la seconde partie tandis que la plasticité se développe en troisième partie. Les observations micrographiques (Fig. 5) montrent que la décohésion interfaciale s'amorce aux pôles des filaments dans l'axe du chargement et précède l'apparition de fissures matricielles.

Comme précédemment, une décohésion interfaciale peut entraîner une nouvelle décohésion au niveau d'un renfort voisin. On notera (Tab. 3) que ces mécanismes d'endommagement sont responsables de la moins bonne tenue mécanique du composite dans le sens transverse [17].

\subsection{Sollicitation cyclique}

Lorsque le chargement est cyclique, les mécanismes d'endommagement dominants sont similaires. Dans le sens longitudinal [18], la surcharge des filaments est accrue par (i) la dégradation progressive du transfert de charge entre renfort et matrice par endommagement interfacial au niveau d'un filament rompu, (ii) l'adoucissement de la matrice par plasticité cyclique qui entraîne une chute de contrainte moyenne dans la matrice et donc une augmentation de la charge moyenne dans le renfort (pour un essai à contrainte imposée). Pour des niveaux de

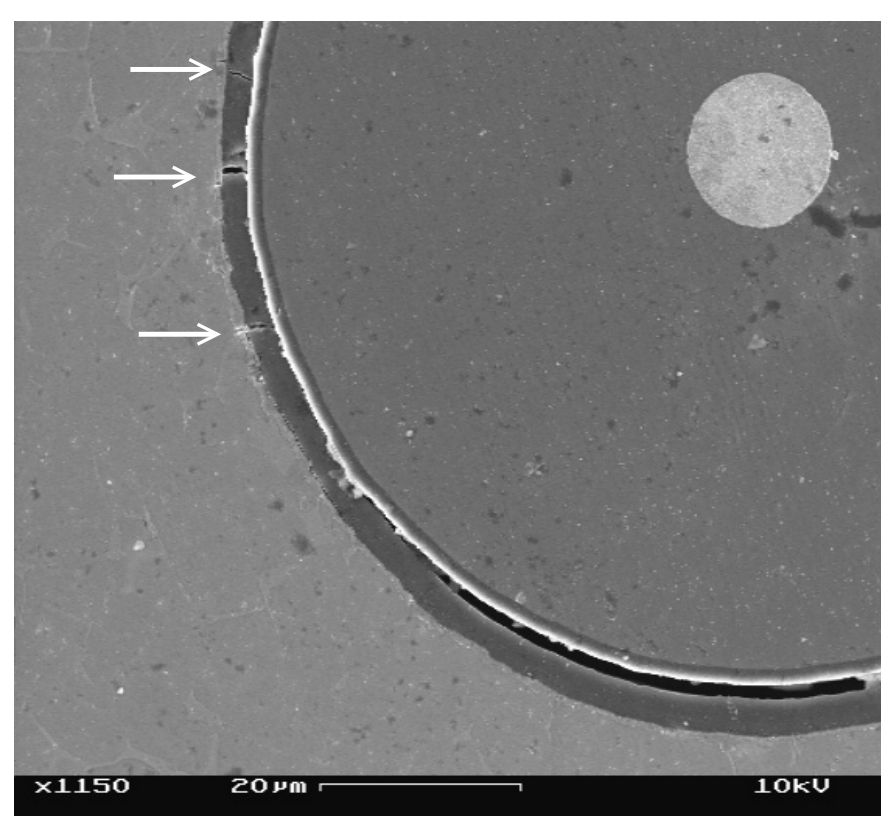

Fig. 5. Observation micrographique de la zone interfaciale sur une éprouvette de composite $\mathrm{SiC} / \mathrm{Ti}$ sollicitée dans la direction transverse (le chargement est appliqué suivant la verticale).

contrainte plus faibles, les ruptures des filaments sont limitées mais des fissures matricielles sont amorcées à partir de ruptures dans la zone de réaction. La déviation de ces fissures matricielles à l'interface renfort/matrice provoque l'établissement d'un régime de fissures pontées par les filaments. Là encore, l'endommagement interfacial sous chargement cyclique surcharge les filaments pontants et favorise la propagation des fissures [19].

\subsection{Influence de la température}

L'influence de la température sur ces mécanismes est non négligeable puisqu'elle favorise la relaxation des contraintes dans la matrice et diminue la contrainte résiduelle de compression axiale dans le renfort. Les chargements thermomécaniques hors phase (fatigue anisotherme) peuvent se révéler particulièrement endommageants pour la matrice lors de la baisse de la température : les contraintes résiduelles viennent alors se superposer au chargement mécanique [20]. Des essais de fluage sous vide ont également permis de confirmer les mécanismes précédents $[21,22]$. La relaxation de la matrice entraîne une surcharge progressive du renfort dans le sens longitudinal ou de l'interface dans le sens transverse. Dans les deux cas, il est possible de mettre en évidence une contrainte seuil correspondant à une durée de vie très longue. En deçà de cette contrainte, la relaxation de la matrice ne permet pas le développement de l'endommagement.

La caractérisation des mécanismes de rupture met en relief le rôle de l'interface renfort/matrice dont l'endommagement conditionne la surcharge des filaments voisins (cas d'une sollicitation longitudinale) ou des interfaces voisines (cas d'une sollicitation transverse). Diverses 
études ont donc été menées afin d'optimiser le comportement interfacial en réduisant les interactions physicochimiques [23, 24] et de caractériser l'interface à l'aide d'essais micromécaniques [25,26].

\section{Analyses micromécaniques}

La détermination des propriétés locales des constituants du composite est une étape préliminaire indispensable à toute démarche multiéchelle. Si les propriétés élastiques paraissent assez simples à appréhender (par des approches inverses par exemple), il n'en est pas de même en ce qui concerne les propriétés interfaciales et viscoplastiques. L'interface est généralement caractérisée en utilisant des essais d'indentation (ou de «push-out») sur filament (pour le comportement tangentiel) et de traction transverse (pour le comportement normal).

\subsection{Comportement tangentiel de l'interface}

Les échantillons de «push-out » sont des lames minces d'épaisseur inférieure à $500 \mu \mathrm{m}$ découpées perpendiculairement à l'axe des renforts. Une charge est appliquée au sommet d'un filament à l'aide d'un indenteur cylindrique instrumenté jusqu'à décohésion complète de l'interface et extraction du filament (Fig. 6) [27]. L'exploitation des résultats (courbe donnant la charge en fonction du déplacement de l'indenteur) est souvent effectuée à l'aide de modèles analytiques unidimensionnels basés sur une notion d'équilibre de contrainte, ou de modèles de type «shear-lag» [28]. En fait, l'utilisation d'une modélisation par éléments finis est indispensable si l'on souhaite remonter à des propriétés intrinsèques correctes [25]. En effet, la phase de fabrication du composite et la découpe de la lame engendrent un champ de contrainte complexe à l'interface renfort/matrice pouvant dans certains cas conduire à un endommagement précoce de l'interface. Un modèle de zone cohésive se révèle bien adapté à la prise en compte du comportement endommageable de l'interface $[29,30]$. Les modélisations montrent que lors d'un essai de «push-out », la décohésion s'amorce et se propage principalement en mode II [22]. Cet essai permet donc d'identifier les termes tangentiels de la loi d'interface. On obtient ainsi l'évaluation de l'énergie de décohésion $G_{\mathrm{II}}^{\mathrm{c}}$ et du coefficient de frottement. Des essais réalisés sur des composites à matrice Ti-6242 montrent que l'énergie de décohésion est très dépendante du type de filament et de revêtement externe employés. On notera par exemple que pour des filaments SCS-6, $G_{\mathrm{II}}^{\mathrm{c}}$ est compris entre $250 \mathrm{Jm}^{-2}$ et $350 \mathrm{Jm}^{-2}$ alors que pour des filaments $\mathrm{SM} 1140+, G_{\mathrm{II}}^{\mathrm{c}}$ vaut environ $11 \mathrm{Jm}^{-2}$.

\subsection{Comportement normal de l'interface}

On utilise dans ce cas des essais de traction transverse. Sous ce type de chargement, le maximum de la contrainte

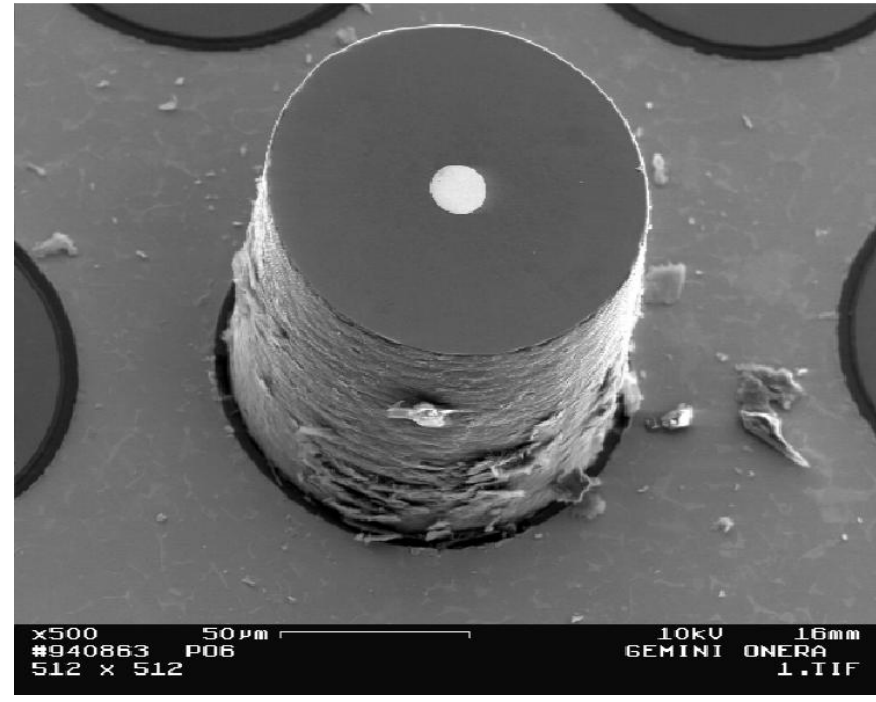

Fig. 6. Essai d'indentation sur filament pour un composite $\mathrm{SiC} / \mathrm{Ti}$.

normale à l'interface est alors situé dans l'axe de chargement [31] et vaut :

$$
\sigma_{r}=K \Sigma+\sigma_{r}^{t h}
$$

où $\Sigma$ est la contrainte transverse macroscopique appliquée, $\sigma_{r}^{t h}$ est la contrainte résiduelle et $K$ un facteur dépendant de la géométrie et des propriétés élastiques des constituants dont la valeur est environ 1,3. Comme noté précédemment, la valeur de la contrainte macroscopique conduisant à la décohésion correspond à la première perte de linéarité de la courbe de traction transverse. La plupart des composites $\mathrm{SiC} / \mathrm{Ti}$ présentent des résistances interfaciales normales assez faibles variant entre $10 \mathrm{MPa}$ et $100 \mathrm{MPa}$ suivant le type de renfort utilisé.

De nombreux auteurs ont noté que les essais travers sur barreaux ne sont géométriquement pas représentatifs du matériau composite dans la pièce finale. En effet, dans les éprouvettes barreaux, les fibres sont débouchantes : il existe au niveau de ce bord libre un état de contrainte singulier pouvant conduire à la rupture prématurée de l'interface [32]. C'est pourquoi, depuis quelques années, une nouvelle géométrie d'éprouvette en forme de croix a été proposée afin de solliciter l'interface dans une zone éloignée d'un bord libre [26,33].

Il est important de noter que ces essais micromécaniques doivent impérativement s'accompagner d'analyses micrographiques afin de corréler le comportement interfacial lors de l'essai micromécanique à celui observé dans le matériau réel pour apprécier la représentativité des essais. On notera par exemple que dans le cas d'un composite SM1140+/Ti-6242, l'interface sollicitée en «push-out » n'est pas la même que celle sollicitée en traction transverse [22]. 


\subsection{Propriétés viscoplastiques de la matrice}

La plupart des études considèrent que le comportement de la matrice dans le composite est identique à celui de l'alliage monolithique. Des essais mécaniques (essais de traction, essais cycliques et essais de fluage) sur éprouvettes d'alliage sont ainsi réalisés à plusieurs températures afin de déterminer les termes de la loi élastoviscoplastique représentant le comportement de la matrice $[13,22,26]$. Cette loi est ensuite utilisée dans des calculs par éléments finis sur une cellule périodique représentant la structure entière du composite.

Cependant, il a récemment été montré, grâce à la technique de diffraction d'électrons rétrodiffusés, que la microstructure de la matrice dans le composite est différente de celle d'un alliage monolithique classique [34]. La matrice in situ présente une texture marquée qui résulte de son mode de fabrication. Dans le cas de l'élaboration par la voie fibre/feuillard, la texture de la matrice est directement héritée de celle du feuillard. La loi de comportement de cette matrice texturée est alors obtenue à l'aide d'une loi de comportement polycristalline qui intègre le comportement du monocristal (identifié à partir d'essais sur de l'alliage isotrope) et l'orientation de chacun des grains dans le polycristal [22].

La connaissance des propriétés des constituants et des mécanismes d'endommagement permet d'envisager la prévision de la contrainte à la rupture du matériau.

\section{Prévision de la contrainte à la rupture}

Dans le cas d'une sollicitation longitudinale, le mécanisme prépondérant de rupture de filaments a incité plusieurs auteurs à transposer l'approche déjà utilisée pour prévoir la contrainte ultime des composites à matrice organique [35]. Afin d'analyser le mécanisme de rupture, on suppose que le transfert de charge filament/matrice provoque au niveau d'un filament rompu, le rechargement du filament qui retrouve la charge de ses voisines à une distance $\delta / 2$ de sa rupture. Un modèle très simple de transfert de charge par cisaillement considère que :

$$
\delta=\frac{R_{f} \sigma_{f}}{2 \tau}
$$

où $R_{f}$ est le rayon de la fibre, $\sigma_{f}$ la contrainte axiale dans le filament et $\tau$ la contrainte de cisaillement interfacial caractéristique de l'interface. À l'intérieur de cette zone de rechargement, les filaments voisins se trouvent surcontraints. Le niveau de surcontrainte est donné par une loi de partage que l'on peut choisir locale en supposant que les filaments voisins d'une rupture et encore intacts se partagent de façon identique toute la charge transférée. La surcontrainte imposée à un filament non rompu est donc fonction du nombre de ruptures avoisinantes. La rupture finale du composite intervient pour une charge appliquée provoquant un phénomène de rupture en cascade des filaments. En général, la statistique de rupture du renfort céramique est décrite par la loi de Weibull. Enfin, l'emploi d'une méthode de Monte-Carlo qui

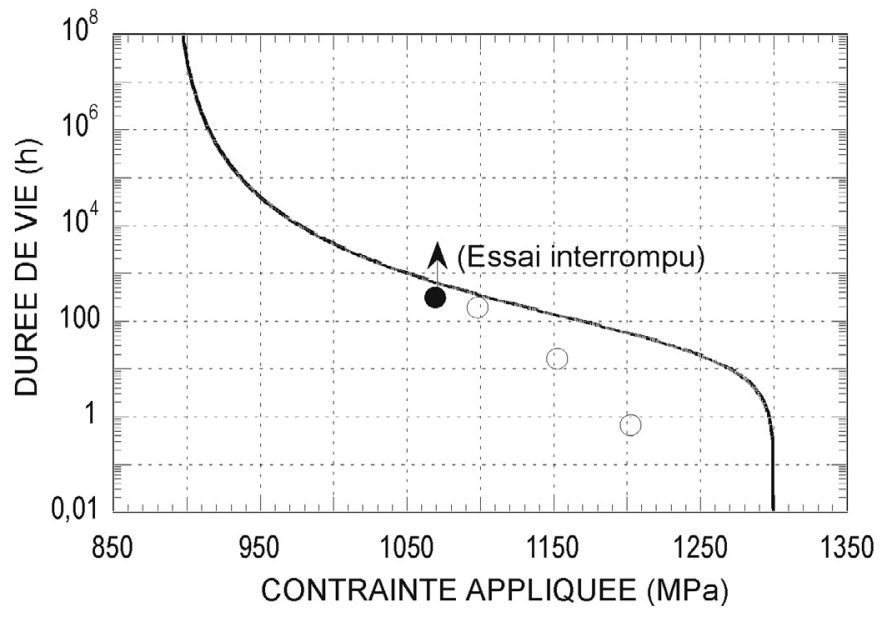

Fig. 7. Comparaison des durées de vie avec les valeurs prédites par un modèle décrivant la rupture des filaments lors d'un essai de fluage longitudinal à $500{ }^{\circ} \mathrm{C}$ sous vide sur un composite SM1140+/Ti-6242.

consiste à découper chaque filament en un certain nombre d'éléments est possible lorsque le nombre de filaments pris en compte reste modéré. Il est ainsi possible d'estimer la contrainte moyenne qu'il faut appliquer au renfort pour provoquer la rupture du composite en tenant compte des interactions entre filaments rompus.

En utilisant des données expérimentales concernant les paramètres de Weibull des filaments ainsi que la contrainte de cisaillement interfacial caractéristique de l'interface, cette approche donne des résultats convenables dans le cas d'un chargement monotone si on prend soin de ne pas négliger la contrainte de compression résiduelle dans le filament [36]. Elle a été étendue pour évaluer la durée de vie du composite sous sollicitation longitudinale en fatigue en introduisant une loi d'usure de l'interface et en fluage en prenant en compte le comportement viscoplastique de la matrice $[18,21]$. À titre d'exemple, la figure 7 compare les valeurs de durée de vie obtenues lors d'essais de fluage avec les prévisions du modèle.

Les simulations indiquent que c'est la formation d'un groupe comprenant un nombre critique de filaments rompus qui est à l'origine de la rupture du composite [37]. Une confirmation expérimentale de l'existence de cet endommagement critique faciliterait l'établissement de critères lors de l'inspection de pièces en composite. On notera que cette approche peut être améliorée en prenant en compte les interactions entre ruptures non coplanaires [38] et en utilisant une meilleure description du comportement interfacial [39].

Lorsque le matériau $\mathrm{SiC} / \mathrm{Ti}$ est soumis à un chargement plus complexe qu'une simple sollicitation longitudinale, il devient nécessaire d'utiliser une stratégie différente en vue du calcul de structure. 


\section{Analyse multiéchelle en vue du calcul de structure}

Les méthodes de changement d'échelle reposent sur les relations de localisation qui permettent de relier la déformation (ou la contrainte) locale à la déformation (ou respectivement la contrainte) macroscopique. Si ce passage est bien établi dans le cadre de l'élasticité, il n'en est pas de même pour les comportements non linéaires.

\subsection{Changement d'échelle dans le cas des matériaux inélastiques}

On peut distinguer deux grandes familles de modèles pour les comportements non linéaires. Une première méthode, développée dans le cadre des polycristaux, consiste à linéariser le comportement local en introduisant des formulations tangentes [40,41], sécantes [42], affines [43] ou du second ordre [44]. Ceci permet ensuite d'utiliser les relations de localisation classiques pour les matériaux linéaires, le calcul des opérateurs de localisation étant dépendant de la méthode choisie. Ce type de modèle est surtout utilisé dans le cadre des polycristaux. Un autre type de modèle repose sur le découpage du volume élémentaire représentatif en $N$ sousvolumes, et sur l'hypothèse de champs de déformation et de contrainte uniformes par sous-volume. On se limitera ici à la méthode de transformation de champ développée par Dvorak dans le cadre des composites à fibres longues [45]. Ce modèle consiste à établir une relation entre la contrainte locale $\sigma_{r}$ dans un sous-volume $r$ et la contrainte macroscopique $\Sigma$ sous la forme :

$$
\sigma_{r}=B_{r}: \Sigma-\sum_{s=1}^{N} F_{r s}: L_{s}: \varepsilon_{s}^{g}
$$

où $B_{r}$ est un tenseur d'ordre 4 appelé tenseur de localisation, $L_{s}$ est le tenseur des raideurs du sous-volume $s$, $\varepsilon_{s}^{g}$ est l'ensemble des déformations inélastiques (plasticité, endommagement par décohésion ou ruptures de fibres) du sous-volume $s$ et $F_{r s}$ est un tenseur traduisant l'influence d'une déformation anélastique du sous-volume $s$ sur la contrainte dans le sous-volume $r$. On notera qu'il existe l'écriture duale en déformation.

\subsection{Stratégie pour le calcul de structure}

Dans le cadre d'un calcul de structure, plusieurs options sont envisageables afin de décrire le comportement macroscopique du composite. On peut choisir d'écrire une loi de comportement macroscopique à l'aide de passages micro-macro. Les relations présentées au paragraphe précédent permettent alors de reconstruire une loi de comportement macroscopique qui garde en mémoire l'échelle microscopique. Dans ce cas, le nombre de sousvolumes doit rester faible pour limiter le nombre de variables internes. Il s'agit d'une méthode « séquencée ».
On peut décider de résoudre les lois de comportement à l'échelle locale. Ceci peut se faire en utilisant une approche à champs moyens (Éq. (3)) ou une approche purement numérique qui substitue, en chaque point de Gauss, la loi de comportement par un calcul sur une cellule élémentaire. Ce dernier type de méthode est désigné sous le terme «éléments finis imbriqués » ou $\mathrm{EF}^{2}$ [46].

\subsection{Calcul de structure à l'aide d'approches multiéchelles}

On se restreint ici à la présentation d'une méthode séquencée, construite à partir d'un découpage en deux sous-volumes $(N=2)$. La loi de comportement macroscopique s'écrit sous sa forme classique :

$$
\Sigma=L^{\text {hom }}:\left(E-E^{g}\right)
$$

avec les relations habituelles entre les deux échelles : $E=\langle\varepsilon\rangle, \Sigma=\langle\sigma\rangle$ et $E^{g}=\left\langle B^{T}: \varepsilon^{g}\right\rangle$, (le symbole $\langle x\rangle$ désigne la moyenne volumique de $x)$. $L^{\text {hom }}$ est le tenseur des raideurs homogénéisé du composite obtenu par une méthode classique [47]. L'expression de $E^{g}$ est donnée par :

$$
E^{g}=\sum_{r=1}^{2} c_{r} B_{r}^{T}:\left[\left(\tilde{L}_{r}^{-1}-L_{r}^{-1}\right): \sigma_{r}+\varepsilon_{r}^{p}+\varepsilon_{r}^{t h}\right]
$$

$\varepsilon_{r}^{p}$ et $\varepsilon_{r}^{t h}$ sont les déformations plastiques et thermiques du sous-volume $r . L_{r}$ et $\tilde{L}_{r}$ sont les tenseurs des raideurs initiaux et endommagés du sous-volume $r$.

En utilisant les équations (3) à (5), il est possible d'écrire un modèle purement macroscopique dont les termes ne dépendent que des propriétés locales des composants (filament, matrice et interface) et des tenseurs de localisation de la matrice $B_{m}$ [22]. L'effet de l'endommagement local (décohésion d'interface et rupture de fibre) est décrite par un modèle d'endommagement à trois variables scalaires (deux pour la décohésion et une pour la rupture de filament). Les lois d'évolution de ces variables découlent des lois interfaciales déterminées précédemment et de l'exploitation du modèle de prévision de durée de vie présenté dans le paragraphe précédent.

Les figures $8 \mathrm{a}$ et $8 \mathrm{~b}$ présentent la comparaison entre des résultats expérimentaux et ceux obtenus avec le modèle présenté. C'est un exemple de tractioncompression transverse à $500{ }^{\circ} \mathrm{C}$ sur un composite SM1140+/Ti-6242. La modélisation prend en compte les contraintes résiduelles de fabrication. Les effets de refermeture de fissures et de désactivation du dommage, difficiles à modéliser avec un modèle classique purement phénoménologique, sont ici bien pris en compte. Le paragraphe suivant présente la modélisation complète d'un anneau aubagé monobloc renforcé par un composite SM1140+/Ti-6242 en utilisant la loi de comportement obtenue à l'aide de cette approche multiéchelle. 


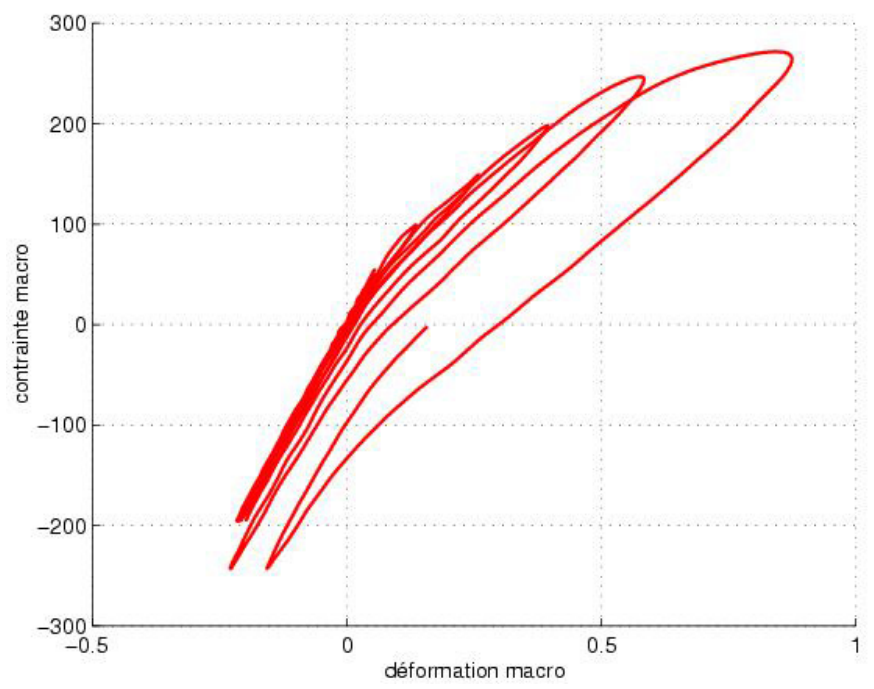

(a)

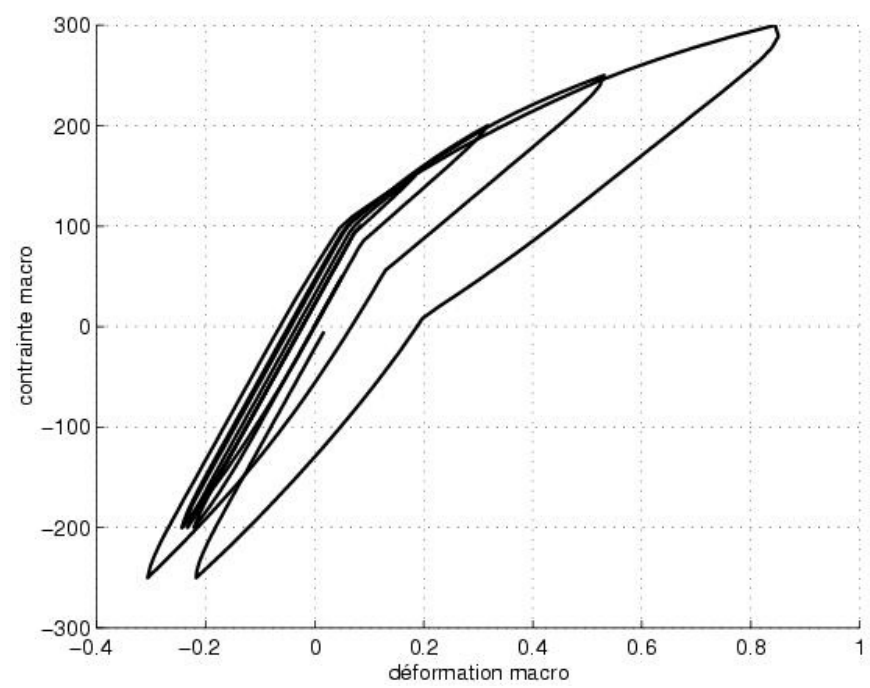

(b)

Fig. 8. (a) Essai de traction cyclique dans le sens transverse à $500{ }^{\circ} \mathrm{C}$ sur un composite SM1140+/Ti-6242. (b) Essai de traction cyclique dans le sens transverse à $500{ }^{\circ} \mathrm{C}$ simulé à l'aide d'une analyse multiéchelle basée sur une méthode séquencée.
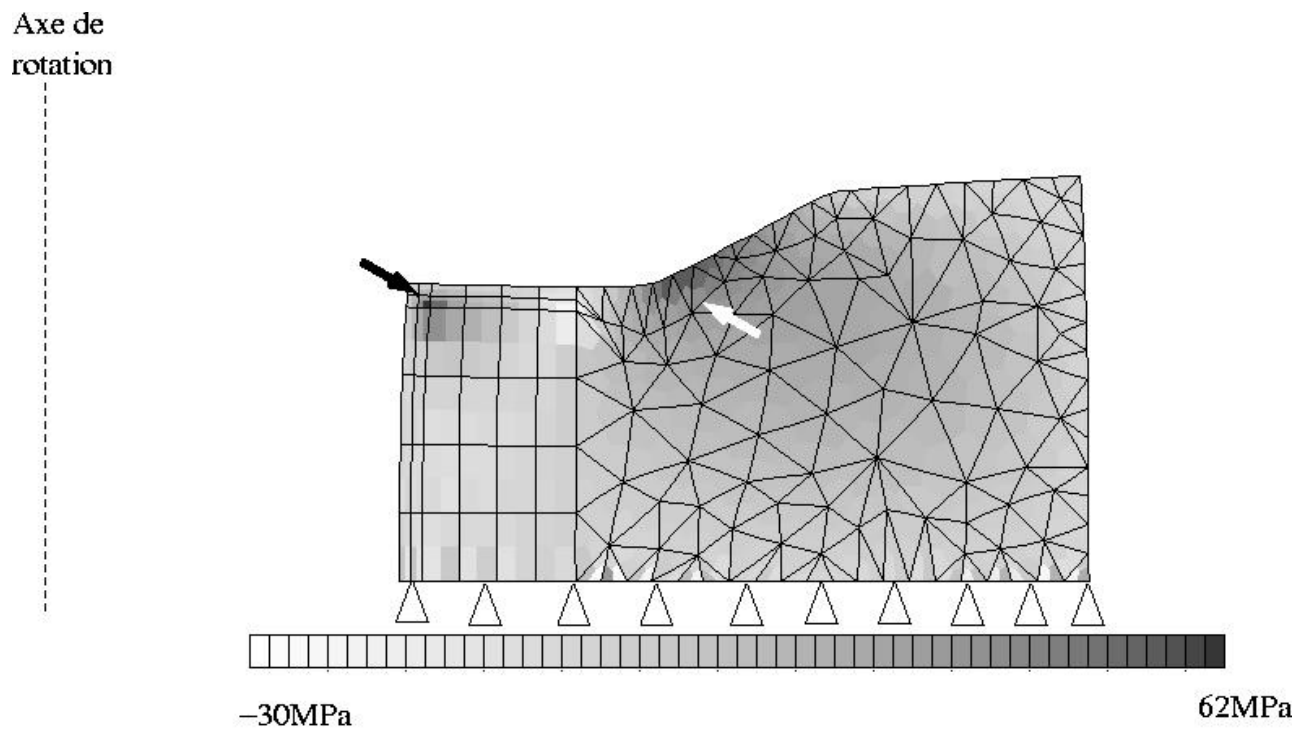

$\sigma_{12}$

Fig. 9. Maillage et conditions aux limites utilisés pour la modélisation de l'anneau aubagé monobloc. La déformation est amplifiée 20 fois et les valeurs de la contrainte de cisaillement dans le plan sont indiquées.

\section{Application à l'anneau aubagé monobloc}

Pour des questions de confidentialité, la modélisation et le maillage ne représentent pas la géométrie réelle de la pièce, mais la forme générale est respectée. Étant données la géométrie et le chargement, le modèle est axisymétrique et seule une moitié de la structure est maillée (le maillage et les conditions aux limites sont présentées sur la Fig. 9). La partie non renforcée est modélisée en utilisant une loi élastoviscoplastique et la partie composite en utilisant la loi présentée au paragraphe précédent. La simulation comporte trois séquences : (i) la phase de fabrication (descente en température de $900{ }^{\circ} \mathrm{C}$ à $25^{\circ} \mathrm{C}$ ), (ii) la phase de mise en température (de $25^{\circ} \mathrm{C}$ à de $900{ }^{\circ} \mathrm{C}$ ) et (iii) la phase de chargement. La vitesse de rotation, choisie arbitrairement, évolue linéairement en fonction du temps (durée du chargement $30 \mathrm{~s}$ ).

\subsection{Résultats}

La figure 9 présente l'état de contrainte de cisaillement dans le plan. Un fort état de contrainte de cisaillement apparaît à l'interface entre le composite et l'alliage de 

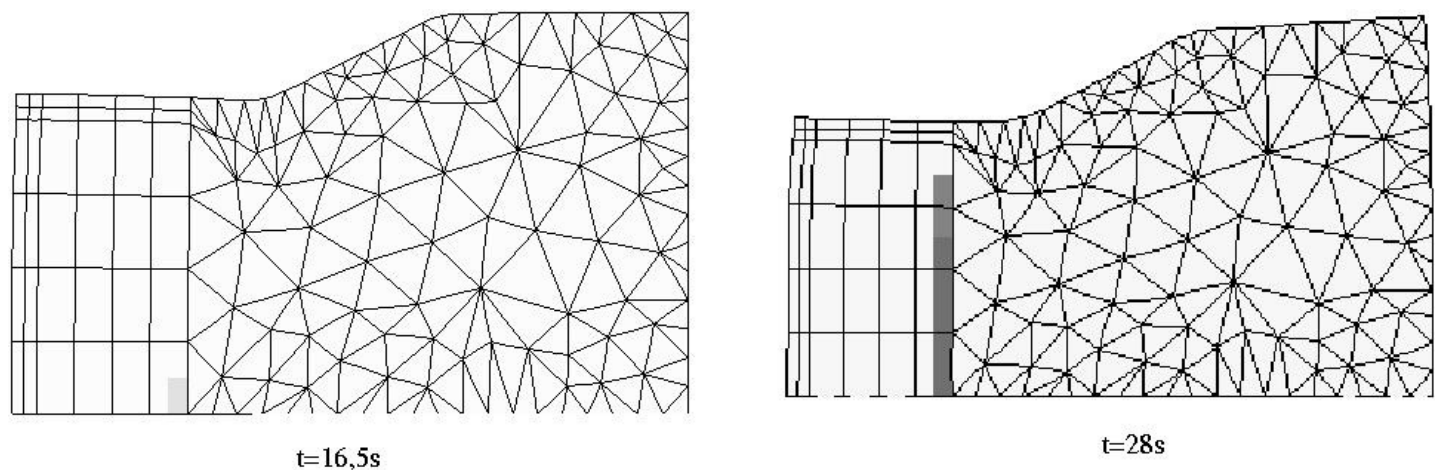

$\mathrm{t}=16,5 \mathrm{~s}$

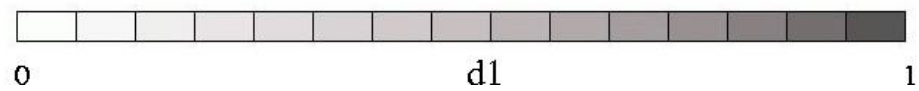

(a)

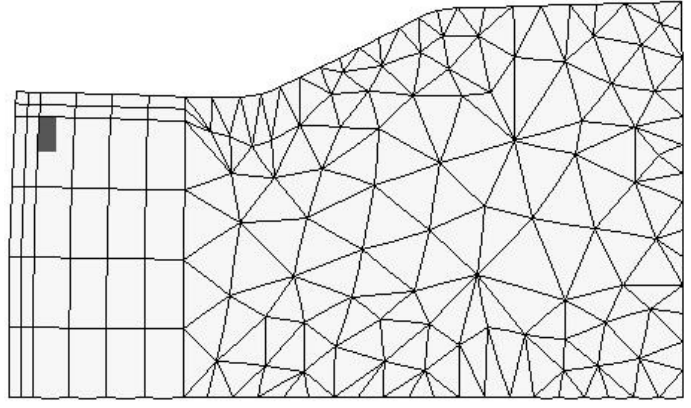

$\mathrm{t}=26.5 \mathrm{~s}$
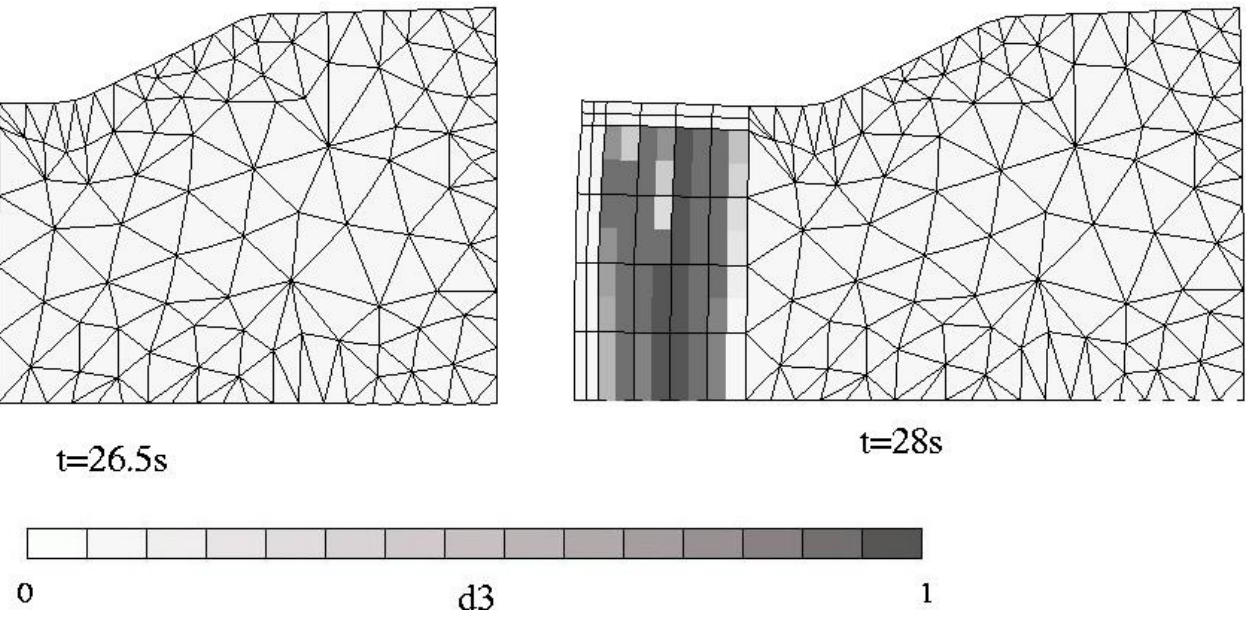

(b)

Fig. 10. (a) Évolution de la variable d'endommagement $d_{1}$ (décohésion filament/matrice). b) Évolution de la variable d'endommagement $d_{3}$ (rupture des filaments).

titane non renforcé (flèche noire sur la figure) et au niveau du raccord entre le disque et l'aube (flèche blanche sur la figure). Ces deux zones sont probablement critiques pour la tenue de la structure.

À l'échelle du volume élémentaire, les quantités locales sont directement disponibles. Ces informations peuvent être très utiles pour appliquer des critères de durée de vie. Notons que les zones critiques, comme les interfaces entre composite et alliage de titane, requièrent une description plus fine des champs locaux. Ces informations peuvent être obtenues à l'aide d'une étape de calcul supplémentaire [47]. Rappelons que les deux principaux mécanismes d'endommagement mis en évidence au sein des composites $\mathrm{SiC} / \mathrm{Ti}$ sont (i) la décohésion de l'interface filament/matrice et (ii) les ruptures de filament. Les figures $10 \mathrm{a}$ et $10 \mathrm{~b}$ montrent les évolutions en cours d'essai de la variable d'endommagement $d_{1}$ qui représente la décohésion de l'interface filament/matrice et de la variable d'endommagement $d_{3}$ qui représente les ruptures de filaments). La décohésion interfaciale débute très tôt en cours d'essai et n'évolue que très lentement dans une petite zone. Les ruptures de fibre apparaissent plus tardivement dans l'essai du côté du rayon interne. Ces ruptures se propagent très rapidement, tout d'abord du même côté, puis dans tout le composite. On peut admettre que lorsque $d_{3}$ vaut 1 dans tout le composite, la structure est rompue.

\subsection{Influence de la fraction volumique de renfort}

L'un des avantages d'une méthode multiéchelle est d'être totalement prédictive. Comme cela a été mentionné plus haut, le composite étudié est encore en phase de développement. Le modèle multiéchelle présenté dans cet article permet de prévoir ainsi l'influence d'une variation des propriétés locales sur le comportement de la structure. Nous présentons ici l'exemple de l'influence de la fraction volumique de filament $\left(V_{f}\right)$.

Deux cas extrêmes sont envisagés : $20 \%$ et $45 \%$ de fraction volumique de renfort. Les résultats pourront être comparés avec le cas de référence de $35 \%$. Tous les paramètres du modèle de comportement sont modifiés par l'intermédiaire du tenseur de localisation $B_{m}$ calculé 
analytiquement. Les paramètres de la loi d'endommagement sont supposés inchangés. Les résultats des calculs montrent que l'augmentation de la fraction volumique conduit à une diminution de la déformation de la structure. À l'échelle filament/matrice, on constate que l'accroissement de $V_{f}$ diminue l'endommagement interfacial. D'un autre coté, lorsque $V_{f}$ croît, les ruptures de filaments apparaissent plus tôt et se propagent plus rapidement. Un compromis doit donc être trouvé entre endommagement longitudinal et transverse. Optimiser la fraction volumique de renfort permet donc d'obtenir un meilleur comportement de la structure à l'échelle globale (déformation du disque) et locale (endommagement).

\section{Conclusion}

L'étude de l'endommagement du matériau composite $\mathrm{SiC} / \mathrm{Ti}$ montre que la rupture du renfort et la rupture de l'interface sont les mécanismes déclenchant la propagation de l'endommagement dans les directions longitudinales et transversales. Les propriétés interfaciales caractéristiques sont obtenues à l'aide d'analyses micromécaniques d'essais d'indentation et de traction transverse. Sous chargement longitudinal, la contrainte à la rupture du composite peut être estimée à l'aide d'un modèle prenant en compte les ruptures multiples des filaments. Dans le cas d'un chargement complexe, des méthodes multiéchelles permettent d'évaluer la loi de comportement du composite.

Remerciements. Les auteurs tiennent à remercier la DGA et la SNECMA pour leur soutien financier.

\section{Références}

[1] S. Mall, T. Nicholas, Titanium matrix composites, S. Mall, T. Nicholas (ed.), Technomic Publications, 1998

[2] J.Y. Guédou, Les matériaux pour turboréacteurs aéronautiques, un défi à la mécanique thermique, $14^{\mathrm{e}}$ Congrès Français de Mécanique, Toulouse, 1999

[3] T.T. Cheng, I.P. Jones, R.A. Shatwell, P. Doorbar, The microstructure of Sigma 1140+ SiC fibres, Mat. Sci. Eng. A260 (1999) 139-145

[4] K. Dyos, R.A. Shatwell, The effect of a weak W/SiC interface on the strength of Sigma silicon carbide monofilament, J. Microsc. 196 (1999) 175-184

[5] R.A. Shatwell, Adhesion of SM1140+ coatings to silicon carbide substrate in Sigma monofilament, Mat. Sci. Techn. 10 (1994) 552-557

[6] M. Peters, J. Kumpfert, C. Leyens, D. Weinem, W.A. Kaysser, Y.T. Lee, Structure and properties of titanium alloys for high temperature applications, Proc. 2nd Pacific Rim international conference on advanced materials and processing, K.S. Shin, J.K. Yoon, S.J. Kim (ed.), The Korean Institute of Metals and Materials, 1995, pp. 2419-2426

[7] Z.X. Guo, B. Derby, Solid-state fabrication and interfaces of fibre reinforced metal matrix composites, Progress in Materials Science 39 (1995) 411-495
[8] Z.X. Guo, N.R.F. Beeley, A novel powder coated fibre preprocessing route to metal-matrix composites, Adv. Eng. Mat. 3 (2001) 223-226

[9] B. Dambrine, J. Feigenblum, M. Garnier, J. Hamburger, Coating of fibres with molten metals, Brevet WO 9937828, EP 931846, FR 2773820, 2002

[10] A. Vassel, Interface considerations in high-temperature titanium metal matrix composites, J. Microsc. 185 (1997) 303-309

[11] U. Ramamurty, F.C. Dary, F.W. Zok, A method for measuring residual strains in fiber-reinforced titanium matrix composites, Acta Mater. 44 (1996) 3397-3406

[12] E. Maire, A. Owen, J.Y. Buffiere, P.J. Withers, A synchroton X-ray study of a $\mathrm{Ti} / \mathrm{SiC}_{\mathrm{f}}$ composite during in situ straining, Acta Mater. 49 (2001) 153-163

[13] L. Baroumes, Comportement des composites à matrice métallique : du processus de fabrication à la tenue en service, Thèse de l'Université Paris VI, 1998

[14] B. Dambrine, M. Hartley, Développement de méthodes d'essais mécaniques pour CMM base titane, AGARD Report 796, 1994

[15] M.P. Thomas, S. Bate, J.G. Robertson, M.R. Winstone, Developement of novel specimens for mechanical testing of fibre reinforced titanium metal matrix composite, Mat. Sci. Techn. 14 (1998) 1009-1014

[16] K. Takashima, K.M. Fox, C. Barney, J.G. Pursell, P. Bowen, Characterisation of acoustic emission signals during fracture and fatigue of $\mathrm{SiC}$ fibre reinforced titanium alloy composites, Mat. Sci. Techn. 12 (1996) 917-922

[17] M.P. Thomas, M.R. Winstone, Transverse tensile behaviour of fibre reinforced titanium metal matrix composites, J. Mater. Sci. 33 (1998) 5499-5508

[18] N. Legrand, Fatigue de composites à matrice métallique base titane à renfort unidirectionnel de fibre $\mathrm{SiC}$, Thèse de l'École nationale supérieure des mines de Paris, 1997

[19] M.N. Tamin, H. Ghonem, Fatigue damage mechanisms of bridging fibers in titanium metal matrix composites, J. Eng. Mat. Technol. 122 (2000) 370-375

[20] S. Hertz-Clémens, Comportement en fatigue anisotherme d'un composite $\mathrm{SiC} / \mathrm{Ti}$, Thèse de l'École nationale supérieure des mines de Paris, 2002

[21] A. Faucon, Étude de quelques mécanismes d'endommagement au sein d'un matériau composite à matrice d'alliage de titane renforcée par des filaments de carbure de silicium, Thèse de l'Université Bordeaux 1, n 2142bis, 1999

[22] N. Carrère, Sur l'analyse multi-échelle des matériaux composites à matrice métallique : application au calcul de structure, Thèse de l'École polytechnique, 2001

[23] K. Debray, Amélioration du couplage fibre-matrice au sein de matériaux composites à matrice d'alliage de titane, Thèse de l'Université Bordeaux 1, n 1268, 1995

[24] J.F. Fromentin, Réalisation et comportement d'interphases à gradient de propriétés-application au matériau composite $\mathrm{SiC} / \mathrm{Ti}$, Thèse de l'Université Bordeaux 1, $\mathrm{n}^{\circ} 1675,1997$

[25] B. Guichet, Identification de la loi de comportement interfacial d'un composite $\mathrm{SiC} / \mathrm{Ti}$, Thèse de l'École centrale de Lyon, 1998

[26] S. Malon, Caractérisation des mécanismes d'endommagement dans les composites à matrice métallique de type $\mathrm{SiC}$, Thèse de l'École nationale supérieure de Cachan, 2000 
[27] J.I. Eldridge, P.K. Brindley, Investigation of interfacial shear strength in a $\mathrm{SiC}$ fibre/Ti-24Al-11Nb composite by a fibre push-out technique, J. Mech. Sc. Letters 8 (1989) 1451-1454

[28] J.W. Hutchinson, H.M. Jensen, Models of fiber debonding and pullout in brittle composites with friction, Mech. Mater. 9 (1990) 139-163

[29] V. Tvergaard, Effect of fibre debonding in a whiskerreinforced metal, Mat. Sci. Eng. A125 (1990) 203-213

[30] J.-L. Chaboche, R. Girard, A. Schaff, Numerical analysis of composite systems by using interphase/interface models, Computational Mech. 20 (1997) 3-11

[31] P.-M. Lesnes, N. Allio, R. Valle, Combined effects of the fibre distribution and of the fiber matrix or interphase matrix transverse modulus ratio on the possible fracture modes of unidirectional composites submitted to a transverse Loading, Acta Metall. Mater. 43 (1995) 4247-4266

[32] E.S. Folias, On the prediction of failure of a fibre/matrix interface of a composite subjected to a transverse tensile load, J. Comp. Mater. 25 (1991) 869-886

[33] D.B. Gundel, B.S. Majumdar, D.B. Miracle, Evaluation of the transverse response of fibre-reinforeced composite using a cross-shaped sample geometry, Scripta Metall. Mater. 33 (1995) 2057-2065

[34] N. Carrere, D. Boivin, R. Valle, A. Vassel, Local texture measurements in a $\mathrm{SiC} / \mathrm{Ti}$ composite manufactured by the foil-fibre-foil technique, Scripta Mater. 44 (2001) $867-72$

[35] F.H. Leroy, Rupture des composites unidirectionnels à fibre de carbone et matrice thermo-durcissable : approche micro-macro, Thèse de l'Université Bordeaux 1, $\mathrm{n}^{\circ}$ 1589, 1996

[36] L. Molliex, Approche micromécanique de la résistance en traction de composites à matrice métallique, Thèse de l'École centrale de Paris, 1995
[37] M. Ibnabdeljalil, W.A. Curtin, Strength and reliability of fiber reinforced composites: localized load sharing and associated size effects, Int. J. Solids Structures 34 (1997) 2649-2668

[38] S.J. Zhou, W.A. Curtin, Failure of fiber composites: a lattice Green function model, Acta Metall. Mater. 43 (1995) 3093-3104

[39] Z. Xia, W.A. Curtin, P.W.M. Peters, Multiscale modeling of failure in metal matrix composites, Acta mater. 49 (2001) 273-287

[40] R. Hill, Continuum micro-mechanics of elastoplastic polycrystals, J. Mech. Phys. Solids 13 (1965) 89-101

[41] J.W. Hutchinson, Bounds and self-consistent estimates for creep of polycrystalline materials, Proc. Roy. Soc. London A348 (1966) 101-107

[42] M. Berveiller, A. Zaoui, An extension of the selfconsistent scheme to plasticity flowing polycrystal, J. Mech. Phys. Solids 6 (1979) 325-344

[43] R. Masson, M. Bornert, P. Suquet, A. Zaoui, An affine formulation for the prediction of the effective properties of non linear composites and polycristals, J. Mech. Phys. Solids 48 (2000) 1203-1227

[44] P. Ponte Castaneda, Exact second order estimates for the effective mechanical properties of non-linear composite materials, J. Mech. Phys. Solids 44 (1996) 827-862

[45] G. Dvorak, Transformation field analysis of inelastic composite material, Proc. Roy. Soc. Lond. A437 (1992) 311-327

[46] F. Feyel, Application du calcul parallèle aux modèles à grand nombre de variables internes, Thèse de l'École nationale supérieure des mines de Paris, 1998

[47] S. Kruch, Comportement global des matériaux composites viscoélastiques, Thèse de l'Université Paris VI, 1988

Retrouvez nos articles sur le site : www.edpsciences.org/meca 